\title{
Awareness about Gynecological Cancers amongst Tribal Females
}

\author{
Neha Jha ${ }^{1}$, Anita H Panot ${ }^{2}$, Upendra Singh ${ }^{3}$
}

${ }^{1}$ Department of Psychiatry, Centre of Excellence in mental Health, ABVIMS \& Dr. RML Hospital, New Delhi, India. ${ }^{2}$ Assistant Professor, Department of Social Work, Nirmala Niketan, Mumbai University, Mumbai, India. ${ }^{3}$ Faculty, Department of Psychiatry, Centre of Excellence in mental Health, ABVIMS \& Dr. RML Hospital, New Delhi, India.

\begin{abstract}
Background: Gender differences are engrained in our culture and are evident when perceiving women's health and ill health. Health issues of women are of great importance in a society as it directly impacts the fabric of family and society. With limited access to education or employment, high illiteracy rates and increasing poverty levels health improvements for women are quite difficult in developing countries including India. There is a significant lack of official data on gynecological cancers further for women from tribal population which is important for advocacy and public health care planning. Aim: Study aimed at assessing awareness of gynecological cancers amongst tribal females in Borivali Taluka of Mumbai, Maharashtra. Methodology: The research is a descriptive study with a cross-sectional research design. 100 tribal female comprised the total sample. Result: Significant lack of awareness about the Gynecological cancers was seen in the tribal respondents of Aarey circle of Borivalli Taluka, Mumbai regarding the symptoms, treatment, and preventive measures of gynecological cancers. Conclusion: It is evident from previous as well as current study several factors are responsible for poor awareness about gynecological cancers among marginalized tribal respondents.
\end{abstract}

Keywords: Tribal health- gynecological cancers- health advocacy

Asian Pac J Cancer Care, 5 (2), 113-118

\section{Introduction}

Healthy living is important for healthy functioning, number of individuals living with health issues due to unawareness. Understanding of health issues can help treatment on time and proper care. There is a significant ignorance among women regarding the gynecological cancers because most of its symptoms are taken less seriously by the women as well as their families because of the traditional beliefs regarding the gynecological issues like vaginal bleeding and pain. The ignorance is somewhat same among the various groups irrespective of their socio-economic background because the way women's gynecological health is perceived in the family irrespective of it being the second biggest cause of mortality among women across the world [1].

Gynecological cancer is any cancer that occurs in a woman's reproductive organs. The gynecological cancers begin in different places within a woman's pelvis. Each cancer of female reproductive organ is unique,
Submission Date: 04/28/2020Ａcceptance Date: 06/17/2020

\section{Corresponding Author:}

Dr. Neha Jha

Department of Psychiatry, Centre of Excellence in mental Health, ABVIMS \& Dr. RML Hospital, New Delhi, India.

Email: neha22.jha@gmail.com 
no awareness about the disease. The access to prevention and treatment facilities are further adding to the treatment gap. Even though cancer screening programs have been made available in all the regional cancer centres and comprehensive cancer care specialty hospitals, it is restricted to only certain population coverage due to limited awareness and resources [4].

The epidemiological studies have pointed out at early age of marriage, multiple sexual partners, multiple pregnancies, poor genital hygiene, malnutrition, use of oral contraceptives, and poor awareness as risk factors. [5]. The first step towards controlling the cancer burden in target population is to know its status in the population and collect information about the relative access to healthcare facilities.

There is no previous study done on the tribal population residing in the city of Mumbai as reaching tribal population due them residing traditionally in the forest part of the city. This is is the pilot study done to assess the awareness about gynecological cancers amongst the tribal females in an urban city order to use the same for the public health advocacy of their major health issues.

Aim

The study aimed at assessing awareness of gynecological cancers amongst tribal females.

\section{Materials and Methods}

Ethical permission was collected from the department of Social Work, Mumbai University. Further permissions was taken from the tribal leaders to conduct the study in respective tribal hamlets and each participant was asked for their consent before any information was collected from them.

The study was a descriptive research. Cross-sectional research design was used and quantitative information was collected from the respondents. A total of 100 tribal female respondents were selected through purposive sampling technique from Borivali, Taluka of Mumbai, Maharashtra. Married females belonging to age group 20-55 years, belonging to a regional tribe, having no physical or psychiatric illness, after giving written consent for the present study were included in the study. Divorced, separated and widowed females were excluded from the study.

\section{Tools}

\section{Socio-demographic Data Sheet}

It was used to collect personal and clinical information of the respondents like - age, education, duration of marriage, substance use, history of physical illness, no. of hospitalization and nature of work etc.

\section{Self prepared interview schedule}

A self prepared interview schedule that was made with the expert from the field had 59 items. It was prepared in English than translated to Marathi language, after that reverse translation done with 6 (six) research expert working in same field, most appropriate questionnaire in Marathi language which can be easily comprehended by the respondents was used for data collection.

\section{Procedure}

At the very beginning ethical permission was taken from the Research and Ethics board of Department of Social Work, Mumbai University. Permission was further taken from the tribal leaders of respective tribal hamlets to conduct the study in their hamlet. Respondents were selected using purposive sampling technique considering inclusion and exclusion. Each participant was explained about the aim and procedure of the study in detail; following which consent were collected from them. Self prepared interview schedule was administered at the very end to collect data. The self prepared interview schedule was administered individually.

\section{Results}

Table 1 describes the distribution of socio-demographic variables of the respondents. Mean age was 32.53 and SD 10.03. Respondents were divided into three age groups, 38\% respondents covered 20-30 years, 32\% were 41-55 years and 30\% respondents were $31-40$ years age group. Majority of the respondents $42 \%$ educated up to higher secondary, comparison to $34 \%$ respondents were completed secondary education, $16 \%$ respondents educated up to primary and $8 \%$ were graduate. Respondents nature of work 36\% were homemaker compare to $24 \%$ were engaged as Vendor, $16 \%$ worked as cook, only $10 \%$ respondents engaged skilled work, labor, fishing and students were $6 \%, 4 \%$ and $4 \%$ respectively.

Table 2 describes the respondents belong various sub division of the tribal community. Total participants comprised 9 tribal communities only one community respondents $20 \%$ due to further sub vision as compared to all other communities were represented $10 \%$ each.

Table 3 shows that related health services availability in participant's community, aganwadi service covered whole area and other only $46 \%$ respondents taken benefits of Pada services. Respondents understanding about cancer very significant they were not much aware about that sever illness like Vaginal Cancer 94\% respondents are not aware in the same way Cervical, Ovarian, Brest and Uterine cancer also $80 \%, 74 \%, 64 \%$ and $62 \%$ respectively not aware.

Table 4 shows that sign and symptoms of cervical cancer among the respondents. Only 54\% respondents were aware about irregular vaginal bleeding, 46\% respondents know that vaginal discharge with unpleasant odor, similarly $22 \%$ were known that pelvic or back pain. Majority of the respondents $96 \%, 94 \%$ and $93 \%$ were not aware about problem defecating, swelling of legs, urinating and pain during intercourse also sign and symptoms of cervical cancer

Table 5 shows that awareness of sign and symptoms of ovarian cancer among the respondents. Only $27 \%$ and $26 \%$ respondents were aware about menstrual changes and abdominal swelling with weight loss respectively one 
Table 1. Shows Distribution of Socio-demographic Variables among the Respondents

\begin{tabular}{lcc}
\hline Variable & Mean $(\mathrm{N}=100)$ & Std. Dev. \\
\hline Age & 32.52 & 10.03 \\
Age Group & Prequency $(\mathrm{N}=100)$ & 38 \\
$20-30$ & 19 & 30 \\
$31-40$ & 15 & 32 \\
$41-55$ & 16 & 16 \\
Education & & 34 \\
Primary & 16 & 42 \\
Secondary & 34 & 8 \\
Higher Secondary & 42 & 6 \\
Graduate & 8 & 16 \\
Nature of Work & & 24 \\
Labour & 6 & 4 \\
Cook / Maid & 16 & 10 \\
Vendor & 24 & 36 \\
Fishing & 4 & 4 \\
Skilled Worker & 10 & 36 \\
Homemaker & 4 & \\
Student & 36 & \\
\hline
\end{tabular}

third $73 \%$ and $74 \%$ were not aware. Similarly maximum respondents $96 \%$ were not aware like frequent bloating and trouble eating also basic sign of ovarian cancer.

Table 6 shows signs and symptoms of gynecological cancers experienced by the respondents. Astounding results reflected that pelvic and Back pain were experienced by $42 \%$ of the respondents while irregular vaginal bleeding was experienced by $36 \%$ of respondents and $38 \%$ reported having changes in menstrual pattern.

A staggering $88 \%$ who experienced these symptoms said that they didn't visit doctors for these symptoms. Reasons cited for the same were that $26 \%$ of respondents considered these symptoms not having a serious health risk, lack of time was also mentioned by $2 \%$ of respondents and 4\% considered this as a normal issue. Symptoms getting cured on its own was belief of $6 \%$ of respondents for not visiting the doctor.

\section{Discussion}

The study was conducted in Aarey area of Borivali taluka of Mumbai region. For the study researcher chose 100 married respondents belonging to 10 Different tribes between the age group 20-55 who residing in different hamlets. Respondents mean Age was 32.52 and 10.03 SD, which divided in three groups. Analyzing the educational qualification of respondents, $8 \%$ were graduate it found that only young age group studying on to higher level. $42 \%$ respondents have studied till higher secondary after that they are engaged in personal work similarly $34 \%$ secondary and $16 \%$ of respondents were educated up to primary level. Research found that most of the respondents which is $36 \%$ were working as homemaker, they primarily worked at home and looked after children and weren't educated while $24 \%$ respondents were working as vendors with their family members. $16 \%$ of respondents worked as cooks or domestic helps for their living while $10 \%$ respondents were skilled worker, $6 \%$ labor work, $4 \%$ were fishing and studying. Notably except fishing, none of the

Table 2. Description of Tribe Community

\begin{tabular}{lcc}
\hline Type of Tribe & & 10 \\
\hline Audh & 10 & 10 \\
Dhoriya & 10 & 10 \\
Dubla & 10 & 10 \\
Katkari & 10 & 10 \\
Kokana & 10 & 20 \\
Koli Mahadev & 20 & 10 \\
Naykada & 10 & 10 \\
Thakkar & 10 & 10 \\
Warli & 10 & 10 \\
\hline
\end{tabular}


Table 3. Responses on Self Prepared Interview Schedule Services and Understanding

\begin{tabular}{llcc}
\hline S. No. & Statements & Yes (\%) & No (\%) \\
\hline 1 & Angarwadi Services (Community Health services ) & 100 & 0 \\
2 & Availability of tribal hamlet Services & 46 & 54 \\
3 & Heard of Cervical Cancer & 20 & 70 \\
4 & Heard of Ovarian Cancer & 26 & 62 \\
5 & Heard of Uterine Cancer & 38 & 94 \\
6 & Heard of Vaginal Cancer & 6 & 64 \\
\hline
\end{tabular}

Table 4. Awareness about Signs and Symptoms of Cervical Cancer

\begin{tabular}{llcc}
\hline S. No. & Signs and Symptoms & Yes (\%) & No (\%) \\
\hline 1 & Irregular vaginal bleeding & 54 & 46 \\
2 & Vaginal Discharge with unpleasant odor & 46 & 54 \\
3 & Pelvic or Back pain & 22 & 78 \\
4 & Pain during intercourse & 7 & 93 \\
5 & Problems urinating & 6 & 94 \\
6 & Problem Defecating & 4 & 96 \\
7 & Swelling of the legs & 4 & 96 \\
\hline
\end{tabular}

Table 5. Awareness about Signs and Symptoms of Ovarian Cancer

\begin{tabular}{llcc}
\hline S. No. & Signs and Symptoms & Yes (\%) & No (\%) \\
1 & Menstrual Changes & 27 & 73 \\
2 & Frequent bloating & 4 & 96 \\
3 & Trouble eating or upset stomach & 4 & 96 \\
4 & Abdominal swelling with weight loss & 26 & 74 \\
\hline
\end{tabular}

tribal women were doing traditional tribal work to sustain a living as it would be difficult sustaining in the city of Mumbai without having work that was considerably paid.

The study suggested that apart from health, education of tribal respondents is neglected. A majority of respondents were young and in the age group between 20-30 years therefore the results of this study are especially significant since the actions taken based on this report will directly affect these respondents when it came to the preventive nature of the Gynecological cancers while the older aged respondents might not be able to benefit from the changes undertaken but can still be educated about the treatment aspect of the disease.

Anganvadi services (Community health services) covered whole area of study but Pada (hamlet) services pertaining to the specific issues of the tribe were not present at grassroot level as it was found that approximately half area, 46\% covered Pada services. Result reflected that respondents that had heard about types of Gynecological cancer, Uterine cancers were known to $38 \%$ as compared to more fatal breast cancer which was $36 \%$ while Vaginal cancer was known only $6 \%$ of respondents. Out of 100

Table 6. Experiencing Signs and Symptoms of Gynecological Cancers

\begin{tabular}{llcc}
\hline S. No. & Signs and Symptoms & Yes (\%) & No (\%) \\
\hline 1 & Irregular vaginal bleeding & 36 & 64 \\
2 & Vaginal Discharge with unpleasant odor & 10 & 90 \\
3 & Pelvic or Back pain & 42 & 58 \\
4 & Pain during intercourse & 14 & 86 \\
5 & Problems during urinating & 38 & 62 \\
6 & Problem Defecating & 3 & 97 \\
7 & Swelling of the legs & 12 & 88 \\
8 & Menstrual Changes & 38 & 62 \\
9 & Frequent bloating / trouble eating/ upset stomach & 18 & 82 \\
10 & Abdominal swelling with weight loss & 24 & 76 \\
11 & Did you visit doctor with any of these symptoms? & 12 & 88 \\
\hline
\end{tabular}


only $20 \%$ of respondents had understanding about cervical cancer whereas this figure shows that $26 \%$ had heard of Ovarian cancer. Early detection of Gynecological cancer is possible if consideration is given to sign and symptoms pertaining especially to cervical cancer [6].

Lack of awareness among respondents which accounts for $80 \%$ who had no knowledge regarding cervical cancer shows an alarming situation and needs to be addressed as it clearly reflects the gap between testing and diagnosis. Cervical cancer is the most common cause of cancer related deaths in developing countries [7]. In India deaths related to cervical cancer is 67,477 out of 122,844 who are diagnosed with this cancer. Most common age developing cancer in respondent's age between 15-44 years, India has high risk of 15 years and above among 432.2 million populations [8].

There is need of addressing the same on policy level that requires advocacy from NGO's and health activists to take this into account to spread awareness among marginalized groups.

Sign and symptoms of cervical cancer among the respondents were found that very severe condition. $54 \%$ respondents were aware about irregular vaginal bleeding, $46 \%$ respondents know that vaginal discharge with unpleasant odor, similarly $22 \%$ were known that pelvic or back pain. Majority of the respondents $96 \%$, $94 \%$ and $93 \%$ were not aware about problem defecating, swelling of legs, urinating and pain during intercourse also sign and symptoms of cervical cancer as focused by Sreedevi et al [9].

Gynecological cancer in India presents a situation that needs to be controlled at the earliest. As reported by the GLOBOCAN 2018, China and India together contributed more than a third of the global cervical burden, with India reporting 97000 cases, and 60000 deaths [1].

Study finding that Ovarian Cancer also have less awareness among the respondents. Researcher has recorded lack of awareness among the respondents about the different forms of gynecological cancers. The most common of gynecological cancer among Indian women which is cervical cancer remains significantly unknown among the respondents. A striking factor study had founded the absence of knowledge among young respondents. When it has been reported by that there is high prevalence of HPV (Human Papilloma Virus) among tribal girls and young women in India which is the biggest risk factor for Cervical cancer [10].

When the researcher studied for awareness of symptoms and asked whether they posed any threat to their health most of the respondents have shown lack of awareness to the disease which these symptoms could lead to. Only irregular vaginal bleeding was considered a threat to health whereas all other symptoms were believed to be normal and something that can heal easily on its own. This clearly reflects the attitude of women towards their reproductive health by taking the symptoms like abdominal cramps and menstrual changes leniently that could be sign of diseases like gynecological cancers.

From the above table shows that abdominal and Back pain were experienced by $42 \%$ of the respondents. Irregular vaginal bleeding was experienced by $36 \%$ of respondents and $44 \%$ came across to changes in menstrual pattern. Total $26 \%$ of respondents considered these symptoms as non-serious and lack of time was also mentioned by $2 \%$ of respondents and $4 \%$ considered this as a normal issue. "Getting cured" on its own was said by $6 \%$ of respondents for not visiting the doctor. Study found that response to the symptoms that the respondents have faced or they were facing, reflected that due to unawareness they were not taking proper treatment which is the reason for poor prognosis. Mainly pelvic and abdominal pain were present in more than $70 \%$ respondents, this could be attributed to other factors not necessary considered as symptoms of cancer in them. Several factors which were holding back the respondents in getting treatment or visit the nearby clinic was in the same line as shown by the study of respondents belonging to lower socio-economic strata and lives in urban area.

In conclusion, lack of awareness was seen in the tribal respondents of Aarey circle of Borivalli Taluka, Mumbai regarding the symptoms, treatment, and preventive measures of gynecological cancers. As it is evident from previous as well as current study multiple factors are responsible for this unawareness among tribal respondents with literacy levels and effective infrastructure in government hospitals being a few of them. Most importantly, aches and menstrual changes are not seen as a symptoms or signs of a disease and hence is followed by negligence that is one of the main reasons affecting the preventive nature of gynecological cancers. Education can be one of the important tools of change in bridging this gap that will help tribal women to achieve better reproductive health. Awareness of tribal respondents belonging to older age group outside the purview of this study need to be studied that researcher due to the limitations of this study the researcher couldn't cover. This study can act for advocating the rights of tribal women's health especially when gynecological cancers have been to factors like lack of awareness and hygiene, and limited access to gynaecologists [11]. The sources of awareness covered an important part of the problem that can be used to design intervention to address the issue . The findings can be used as means for advocacy to put the light on the various aspects of the gynecological cancers that currently remains unaddressed for the marginal community of tribal women.

\section{Data Availability}

Data is data available on request through ethics committee of College of Social Work, Nirmala Niketan, Mumbai University, Mumbai, India available at the email address of nn@cswnn.edu.in

\section{Conflicts of Interest}

The study represents no conflict of interest which includes financial relationships, personal relationships or rivalries, academic competition, and intellectual beliefs.

\section{Funding Statement}

No funding was secured for the study. 


\section{Acknowledgments}

Dr Rama Joshi, Director, Fortis Memorial Research Institute, Gurugram, India and Head of the Department of Gynecology Oncology was the key expert in designing the tool and providing her expertise throughout the study.

\section{References}

1. Bray F, Ferlay J, Soerjomataram I, Siegel RL, Torre LA, Jemal A. Global cancer statistics 2018: GLOBOCAN estimates of incidence and mortality worldwide for 36 cancers in 185 countries. CA: A Cancer Journal for Clinicians. 2018 09 12;68(6):394-424. https://doi.org/10.3322/caac.21492

2. Uma Devi K. Current status of gynecological cancer care in India. Journal of Gynecologic Oncology. 2009;20(2):77. https://doi.org/10.3802/jgo.2009.20.2.77

3. "International Agency For Research On Cancer: Latest Global Data". 2018. Iarc.Fr. https://www.iarc.fr/wp-content/ uploads/2018/09/pr263_E.pdf.

4. Consolidated report of hospital based cancer registries 2001-3, national cancer registry program. New Delhi: Indian Council of Medical Research; 2007. .

5. Maheshwari A, Kumar N, Mahantshetty U. Gynecological cancers: A summary of published Indian data. South Asian Journal of Cancer. 2016;5(3):112. https://doi. org/10.4103/2278-330x.187575

6. Aswathy S, Reshma J, Avani D. Epidemiology of cervical cancer with special focus\&nbsp;on India. International Journal of Women's Health. 2015 04;:405. https://doi. org/10.2147/ijwh.s50001

7. Denny L. "Cervical cancer: prevention and treatment." . Discovery medicine . 2012;14(75):125-31.

8. ICO Information Centre on HPV and cancer (Summary Report 2014-08-22).Human Papillomavirus and Related Diseases in India. 2014.

9. Aswathy S, Reshma J, Avani D. Epidemiology of cervical cancer with special focus\&nbsp;on India. International Journal of Women's Health. 2015 04;:405. https://doi. org/10.2147/ijwh.s50001

10. Travasso C. Prevalence of HPV is high among Indian tribal girls and young women, study finds. BMJ. 201505 18;350(may18 6):h2692-h2692. https://doi.org/10.1136/ bmj.h2692

11. Dey S, Pahwa P, Mishra A, Govil J, Dhillon PK. Reproductive Tract infections and Premalignant Lesions of Cervix: Evidence from Women Presenting at the Cancer Detection Centre of the Indian Cancer Society, Delhi, 2000-2012. The Journal of Obstetrics and Gynecology of India. 2016 03 11;66(S1):441-451. https://doi.org/10.1007/s13224015-0819-1.

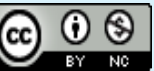

This work is licensed under a Creative Commons AttributionNon Commercial 4.0 International License. 